\title{
A method for the automatic reconstruction of fetal cardiac signals from magnetocardiographic recordings
}

\author{
D Mantini ${ }^{1}$, G Alleva ${ }^{2}$ and S Comani ${ }^{2,3}$ \\ ${ }^{1}$ Department of Informatics and Automation Engineering, Marche Polytechnic University, \\ Ancona, Italy \\ 2 Department of Clinical Sciences and Bio-imaging, Chieti University, Chieti, Italy \\ 3 ITAB-Institute of Advanced Biomedical Technologies, University Foundation \\ “G. D’Annunzio", Chieti University, Chieti, Italy \\ E-mail: comani@itab.unich.it
}

Received 6 May 2005, in final form 26 July 2005

Published 28 September 2005

Online at stacks.iop.org/PMB/50/4763

\begin{abstract}
Fetal magnetocardiography (fMCG) allows monitoring the fetal heart function through algorithms able to retrieve the fetal cardiac signal, but no standardized automatic model has become available so far. In this paper, we describe an automatic method that restores the fetal cardiac trace from fMCG recordings by means of a weighted summation of fetal components separated with independent component analysis (ICA) and identified through dedicated algorithms that analyse the frequency content and temporal structure of each source signal. Multichannel fMCG datasets of 66 healthy and 4 arrhythmic fetuses were used to validate the automatic method with respect to a classical procedure requiring the manual classification of fetal components by an expert investigator. ICA was run with input clusters of different dimensions to simulate various MCG systems. Detection rates, true negative and false positive component categorization, QRS amplitude, standard deviation and signal-to-noise ratio of reconstructed fetal signals, and real and per cent QRS differences between paired fetal traces retrieved automatically and manually were calculated to quantify the performances of the automatic method. Its robustness and reliability, particularly evident with the use of large input clusters, might increase the diagnostic role of fMCG during the prenatal period.
\end{abstract}

\section{Introduction}

Since Kariniemi et al first performed a fMCG in 1974 (Kariniemi et al 1974), fetal magnetocardiography (fMCG) has been reported as a noninvasive technique useful for the prenatal assessment of fetal heart function and fetal well-being, and in particular for the investigation of fetal arrhythmias, congenital heart defects and fetal cardiac hypertrophy 
(Hosono et al 2002, Leeuwen et al 1999, van Leeuwen et al 2004, Kandori et al 2003, Wakai et al 2003, Comani et al 2004c, Kähler et al 2001, Grimm et al 2003, Horigome et al 2001, Lowery et al 2003, Stinstra et al 2002, Li et al 2004). In the last decade, fMCG has been shown to be superior to trans-abdominal fetal ECG, in particular for examinations performed during the second half of gestation; in fact, fetal ECG has a relatively high failure rate because of the insulating properties of the vernix caseosa, whereas fMCG remains unaffected (Lewis 2003, Taylor et al 2003, Stinstra and Peters 2002, Wakai et al 2000). In a recent study, Wakai indicated fMCG as an easy and outstanding method for the antenatal surveillance of fetal well-being, demonstrating that fMCG can also be used to determine fetal trunk movements and to assess fetal neurodevelopment (Wakai 2004).

FMCG recordings are linear mixtures of signals related to fetal and maternal cardiac activity, and to environmental magnetic noise; therefore, the compulsory pre-requisite for a clinically relevant use of fMCG is the successful separation of high-quality fetal traces, which is not easy to achieve because the peak-to-peak amplitude of fetal signals is generally one order of magnitude smaller than that of maternal ones, and even smaller than noise during early gestation. Moreover, occurrences such as unfavorable fetal orientations and/or simultaneous maternal and fetal ventricular depolarization make the separation of signals more difficult.

Several mathematical procedures have been proposed to extract reliable fetal signals such as the correlation method (Abraham-Fuchs et al 1990), an eigenvector based spatial filtering (Chen et al 2001), a projector operator method (Vrba et al 2004) and an adaptive maternal beat subtraction procedure (Comani et al 2004e). However, fetal traces may be affected by maternal and/or noise contamination, and RR interval series are generally corrected for missing fetal $\mathrm{R}$ peaks after a visual inspection, and the signal-to-noise ratio (SNR) of $\mathrm{P}$ and $\mathrm{T}$ waves is improved through signal averaging. Though, averaging implies the loss of information on cardiac waves variability within a single fetus, and it may induce a distortion of the weak and slow $\mathrm{P}$ and $\mathrm{T}$ waves because of the asymmetry in the alignment point ( $\mathrm{R}$ peak) with respect to the cardiac cycle extremes (Comani et al 2005a).

As an alternative, independent component analysis (ICA) can be used to separate dependable fetal cardiac signals from fMCG, as we already demonstrated for healthy and arrhythmic fetuses in both singleton and twin pregnancy (Comani et al 2004c, 2004d, 2004e, Comani et al 2005b); problems such as the simultaneity of fetal and maternal QRS complexes or the fetal signal weakness can be efficiently handled with ICA. Although ICA has already been employed to process different kinds of biomedical signals (James and Hesse 2005, Esposito et al 2005, Zhou and Gotman 2005, Pichler and Sowa 2005, Tran et al 2004, Saidi et al 2004, Nakamura et al 2004, Salustri and Kronberg 2004), its use to extract fetal signals from fMCG recordings has required the investigator's identification of fetal components so far, whereas only an automatic procedure might have a real clinical impact.

Indeed, several problems need to be solved in order to implement a dependable ICA-based automatic method to process fMCG: first, a large number of input recordings must be used to obtain a stable separation of all source signals, each with a sufficient signal-to-noise ratio (SNR) (Comani et al 2004a, Mantini et al 2004); second, there is no a priori information on the total number of independent sources nor on the number of components related to fetal cardiac activity; finally, it is not possible to predict the order with which all components will be sorted.

In this paper, we describe a method for the automatic retrieval of fetal cardiac signals from multichannel fMCG. ICA was used to separate independent source signals, which were categorized with dedicated algorithms that analyse the frequency content and the time structure of each component; the correct amplitude and polarity of fetal cardiac signals were restored using the information embedded in input traces. The procedure was integrated in the software 
suite for the analysis of MCG data recorded with different devices, which is operational in our laboratory (Comani et al 2004b). The performances of the proposed method were tested on $66 \mathrm{fMCG}$ datasets of normal fetuses of increasing gestational age, and on four datasets of arrhythmic fetuses recorded towards late gestation; two input cluster configurations were used to simulate the set-up of different multi-channel MCG devices, with the aim of assessing the dependence of the automatic method on the number and quality of input traces. The outcomes of the proposed model were compared with the results of the classical approach that uses the manual categorization of components.

\section{Materials and methods}

\subsection{Acquisition system and recorded signals}

Seventy fMCG datasets related to singleton pregnancies (66 healthy fetuses, gestational age 22-37 weeks; four arrhythmic fetuses, gestational age 30-37 weeks) were used for the present study, which had been approved by the Ethics Committee of our University. All volunteers signed a written informed consent before undergoing fMCG examination, which was performed with a flat multi-channel dc-SQUID system operating in a shielded room and having 55 sensors homogeneously arranged on a circular surface of $230 \mathrm{~mm}$ diameter (Della Penna et al 2000). Each acquisition lasted from 5 to $10 \mathrm{~min}$ and provided a dataset of 55 simultaneous fMCG traces recorded in the bandwidth $0.016-250 \mathrm{~Hz}$ at $1 \mathrm{kHz}$ sampling frequency.

\subsection{FMCG signal processing}

FMCG recordings were pre-processed with a Chebyshev II-type filter (band-pass $0.4 \mathrm{~Hz}-$ $150.0 \mathrm{~Hz}$ ) for background noise reduction, and a notch filter at $50 \mathrm{~Hz}$ for power line disturbance rejection.

Independent component analysis (ICA) was used to process fMCG datasets (Comon 1994). The general ICA model assumes that a set of observations of $n$ random variables $\left[x_{1}(t), \ldots, x_{n}(t)\right]^{T}$ is generated as an instantaneous linear mixture of $m$ independent source signal components $\left[s_{1}(t), \ldots, s_{m}(t)\right]^{T}$. The ICA linear expansion

$$
x(t)=A s(t)
$$

was fitted to the data vector $x(t)$, where $A$ is a constant $[n \times m]$ matrix called the mixing matrix. Since $A$ is a full-rank matrix, the number of independent components $(m)$ must be at most equal to the number of mixtures $(n)$.

The ICA problem can be solved using the information contained in $x(t)$ : an $[m \times n]$ matrix $W$, called the un-mixing matrix, and an $m$-dimensional vector $y(t)=W x(t)$ are retrieved; under the hypothesis that the source signals are independent, a good estimate of $s(t)$ is given by a vector $y(t)$ that can be found minimizing the average mutual information

$$
I=\int p_{y}(u) \log \frac{p_{y}(u)}{\prod_{i=1}^{n} p_{y i}(u)} \mathrm{d} u .
$$

where $p_{y}$ is the probability distribution function associated with $y(t)$, and $p_{y i}$ is the probability distribution function associated with the $i$ th component of $y(t)$.

The hypothesis on the independence of source signals was satisfied in the case of fMCG datasets, because the maternal and fetal hearts produce independent signals that are linearly mixed in fMCG recordings (Comani et al 2004a, 2004e). 
The fixed-point ICA algorithm (FastICA) was used (Hyvärinen 1999, Hyvärinen and Oja 2000); it minimizes mutual information using the normalized differential entropy or negentropy (Comon 1994). The entropy of a random variable is related to the information contained in its observations: the more random the variable, the larger its entropy. In the case of a random vector $y(t)$ with density $p_{y}($.$) , entropy H$ can be defined as

$$
H(y)=-\int p_{y} \log \left(p_{y}\right) \mathrm{d} y .
$$

As it is known from information theory, the entropy of a Gaussian variable is the largest among the entropies of all random variables of equal variance; therefore, negentropy $J$ is defined as

$$
J(y)=H\left(y_{\text {Gauss }}\right)-H(y)
$$

where $y_{\text {Gauss }}$ is a Gaussian random vector with the same correlation matrix of $y$. Negentropy can also be interpreted as a measure of non-Gaussianity; in the case of uncorrelated variables, the relation between mutual information and negentropy is

$$
I=J(y)-\sum_{i=1}^{n} J\left(y_{i}\right) .
$$

As a result, finding an invertible transformation $W$ that minimizes mutual information is equivalent to finding directions for which negentropy is maximized; FastICA uses simple estimates of negentropy based on the maximum entropy principle (Hyvärinen 1998). This approximation requires the use of an appropriate non-linear function $G$ to implement the learning rule for the neural network. According to the FastICA theory, the one-unit cost function

$$
J_{G}(w)=\left[E\left\{G\left(w^{T} x\right)\right\}-E\{G(v)\}\right]^{2}
$$

was used to find one independent component; $w$ was an $m$-dimensional vector such as $E\left\{\left(w^{T} x\right)^{2}\right\}=1$, and $v$ was a Gaussian variable with zero mean and unit variance. This approach required no a priori knowledge on the total number of independent components, because they are estimated one by one. However, function $G$ had to be carefully chosen to obtain a good approximation of negentropy. For fMCG application, the following function $G($.$) and the related derivative g($.$) ensured great robustness and fast convergence:$

$$
G(u)=\mathrm{e}^{\frac{u^{2}}{2}}, \quad g(u)=u \mathrm{e}^{\frac{u^{2}}{2}} .
$$

The first independent component $y_{1}=w^{T x}$ was retrieved using an optimization algorithm, called fixed-point algorithm, which maximizes the cost function $J_{G}(w)$ using two steps:

$$
\begin{aligned}
& w^{+}=E\left\{x g\left(w^{T} x\right)\right\}-E\left\{g^{\prime}\left(w^{T} x\right)\right\} w \\
& w^{*}=w^{+} /\left\|w^{+}\right\|
\end{aligned}
$$

where $w^{*}$ denotes the new value of $w$, which is iteratively estimated until convergence.

At the end of the described procedure, one independent component is separated. Other components can be found with a deflation scheme that sequentially uses the same method on subspaces orthogonal to the previously recovered vectors (Hyvärinen 1999, Hyvärinen and Oja 2000).

In general, one fetal component (seldom two) can be expected; very rarely three fetal components are separated. We demonstrated that six independent components (ICs) are sufficient to separate all source signals, and to reconstruct comprehensive fetal traces (Comani et al 2004a). 


\subsection{Automatic classification of components}

A PC with $2 \mathrm{GHz}$ Pentium IV ${ }^{\mathrm{TM}}$ processor, $512 \mathrm{MHz}$ RAM and the MATLAB interactive programming environment (MathWorks, USA, version 6.5), powered by vectorial computing and visualization tools, were used to implement the automatic classification algorithms, which analysed the frequency content and temporal structure of components, as described in the following sections.

2.3.1. Frequency domain analysis of ICs. Information on the frequency content of components was used to identify background noise; maternal and fetal components that were too noisy to be employed for the reconstruction of cardiac signals were also categorized as noise.

For each independent component, the power $P_{\mathrm{LF}}$ of the spectral band between 10 and $40 \mathrm{~Hz}$, where ventricular depolarization frequencies are typically included, and the power $P_{\mathrm{HF}}$ of the spectral band between 110 and $140 \mathrm{~Hz}$, where frequencies due to cardiac activity are negligible, were calculated (Stinstra et al 2000).

Cardiac components usually have $P_{\mathrm{LF}} \gg P_{\mathrm{HF}}$, although real cases may present residual noise contamination; conversely, Gaussian noise components have $P_{\mathrm{LF}}$ almost equal to $P_{\mathrm{HF}}$. As a result, the ratio $P_{\mathrm{LF}} / P_{\mathrm{HF}}$ is close to unity for noise components and noisy cardiac components, which can be categorized using the following condition

$$
\frac{P_{\mathrm{LF}}}{P_{\mathrm{HF}}} \leqslant h,
$$

where $h$ is a parametric threshold that was set equal to 3 ; with this parameter value, components satisfying equation (9) correspond to noise or to cardiac components with a SNR smaller than $5 \mathrm{~dB}$. The remaining components, related to cardiac activity, were left for further categorization as maternal or fetal. To select cardiac components with a higher SNR, it is sufficient to increase the value of the threshold $h$.

2.3.2. Time domain analysis of ICs. The components not satisfying equation (9) were analysed in the time domain in order to be classified as maternal or fetal. For each component, the number of QRS complexes was found as the number $N$ of times when a function $z(t)=s(t) \dot{s}(t)$ was larger than $b(t)$, which was a threshold set equal to half the average peak-to-peak intensity of $z(t)$ for subsequent windows of $1200 \mathrm{~ms}$. The average heart rate $\mathrm{HR}_{\mathrm{ave}}$, given in beats per minute (bpm), was then calculated as

$$
\mathrm{HR}_{\mathrm{ave}}=\frac{k N}{\sum_{i=1}^{N-1}\left(t_{i+1}-t_{i}\right)},
$$

When the instants $t_{i}$, corresponding to the peak ventricular depolarization ( $\mathrm{R}$ wave), were expressed in ms, $k$ was equal to 60000 .

Maternal and fetal components were differentiated on the basis of the value obtained for $\mathrm{HR}_{\mathrm{ave}}$. Maternal and fetal heart rates rarely overlap; therefore, components with $\mathrm{HR}_{\mathrm{ave}}$ ranging from 50 to $120 \mathrm{bpm}$ were classified as maternal, whereas components with $\mathrm{HR}_{\mathrm{ave}}$ greater than $120 \mathrm{bpm}$ were classified as fetal. In the case of maternal or fetal arrhythmias, those ranges may be tailored to the current condition.

This procedure also permitted the classification of non-Gaussian noise components, such as baseline shifts, because the calculated $\mathrm{HR}_{\mathrm{ave}}$ was generally lower than $5 \mathrm{bpm}$ in those cases, very far from any possible maternal or fetal cardiac rhythm. 


\subsection{Fetal signal reconstruction}

One fetal component was usually identified from each fMCG dataset, and the fetal trace $f_{\mathrm{IC}}(t)$ simply corresponded to it. Sometime two, and very rarely three fetal components were detected; in those cases, $f_{\mathrm{IC}}(t)$ was the weighted summation in time of all fetal components, with weights derived from the mixing matrix A (Comani et al 2004e). The correct intensity and polarity of the fetal signal $f(t)$ were restored with scaling coefficients, according to

$$
f(t)=f_{\mathrm{IC}}(t) \frac{b_{\mathrm{ave}}}{d_{\mathrm{IC}}} .
$$

In equation (11), $b_{\text {ave }}$ represents the peak-to-peak amplitude of the averaged fetal cycle, which was calculated from fMCG recordings using the instants corresponding to R peaks in $f_{\mathrm{IC}}(t)$ as trigger points. On the other hand, $d_{\mathrm{IC}}$ is the peak-to-peak amplitude of the average beat in $f_{\mathrm{IC}}(t)$. Since coefficients $b_{\text {ave }}$ and $d_{\mathrm{IC}}$ may be either positive or negative, the correct trace polarity was restored together with the appropriate amplitude (Comani et al 2004d). When necessary, the SNR of $f(t)$ could be further improved with a smoothing procedure based on a Kaiser filter (Mitra and Kaiser 1993) working with a moving window that preserves $1 \mathrm{~ms}$ resolution. The smoothing factor $\sigma$ was defined as the number of samples in the moving window and expressed in ms. Typical $\sigma$ values for $\mathrm{P}$ and $\mathrm{T}$ waves, which are weak signals usually more affected by noise, were of the order of $30 \mathrm{~ms}$, whereas no smoothing of QRS complexes was performed.

\subsection{Validation of the automatic method}

In previous studies, we demonstrated that a stable and repeatable ICA output could be obtained using input clusters with a minimum number of 19 fMCG recordings (observations $x_{j}$ ); however, this lower limit may be different, depending on the SNR of fMCG datasets (Comani et al 2004a, Mantini et al 2004).

Since the number and spatial distribution of sensors may significantly change among MCG devices, for each fMCG dataset we separated ICs using the entire cluster of 55 recordings and nine clusters of 19 recordings each (Comani et al 2004a, 2004d, Mantini et al 2004). The nine clusters were homogeneously distributed over the sensing plane and partially overlapped; one cluster was centred on the recording area, while the others were peripheral and rotated by $45^{\circ}$ with respect to the adjacent ones. Through this approach, we wanted to simulate different realistic conditions and to evaluate how much the performances of the proposed method could depend on the sensor array configuration and its relative position with respect to the fetal heart.

The validation of the proposed method included the comparison of the outcomes of the automatic and manual procedures in relation to (i) the categorization of independent components and (ii) the characteristics of the fetal traces retrieved using the selected fetal components. The validation procedure was performed for the results obtained with clusters of 55 and 19 recordings.

2.5.1. ICs categorization. The manual classification of all extracted components was performed by an expert investigator for each dataset and for each sensor configuration; three signal categories (fetal cardiac activity, maternal cardiac activity and noise) were used, and the number $N_{\operatorname{man}}$ of components belonging to each category was calculated.

Then, the automatic procedure was used, and the number $N_{\text {tot }}$ of components classified for each signal category was obtained; the number $N_{\text {corr }}$ of components that were correctly identified was evaluated comparing $N_{\text {tot }}$ with $N_{\text {man }}$. 
For each signal category, two numerical indices were used to quantify the performance of the automatic method with respect to the investigator's categorization: a first index was the percentage of missed independent components (MIC), which represented the number of components that were not classified (true negatives); it was defined as

$$
\mathrm{MIC}=100\left[\frac{N_{\text {man }}-N_{\text {corr }}}{N_{\text {man }}}\right]
$$

with $N_{\text {corr }}<N_{\text {man }}$.

A second index was the percentage of incorrectly classified independent components (ICIC), which corresponded to false positives and was given by

$$
\text { ICIC }=100\left[\frac{N_{\text {tot }}-N_{\text {corr }}}{N_{\text {man }}}\right]
$$

with $N_{\text {tot }}>N_{\text {corr }}$.

Total values of $N_{\text {man }}, N_{\text {tot }}, N_{\text {corr }}$, MIC and ICIC were calculated for five classes of increasing fetal age, producing summary tables on the performance of the automatic method in function of gestational age and cluster dimensions.

2.5.2. Fetal signals characteristics. The general performance of the automatic method was estimated comparing the number and quality of restored fetal signals with those reconstructed manually. The following quantities were calculated for the traces retrieved automatically and manually: average QRS amplitudes and related standard deviations, and average SNR. The average figures provided for gestational periods are derived from the analysis of single fetuses, for each of which the real difference between the average QRS amplitudes, given in fT, of the traces reconstructed with the two methods was calculated. The ranges of real QRS differences were obtained from the minimum and maximum differences detected among all fetuses in a group. The average per cent figures were obtained from real QRS differences normalized to the manual QRS amplitude estimate.

\section{Results}

Examples of fMCG recordings of three healthy fetuses and one arrhythmic fetus are given in figure 1, while figure 2 shows the intermediate output of the automatic method, corresponding to the separation and classification of ICs, obtained from the datasets of the healthy fetuses shown in figure 1; for each fetus, the outputs obtained using the entire dataset of 55 recordings and a 19-channel cluster are shown, with the automatic classification indicated aside. The fetal signals reconstructed for those fetuses using the automatic and manual procedures can be compared in figure 3, where the correct signal amplitude and polarity are restored. It is worth noting the increase of signal intensity with growing gestational age, in accordance with the increment of fetal heart mass along pregnancy. Those examples clearly demonstrate that the performance of the automatic procedure improved along gestation and with larger input clusters, which provided signals with a higher SNR and, consequently, better-defined morphology.

Figure 4 shows the ICs separated with two different clusters from the dataset of the arrhythmic fetus shown in figure 1, as well as the related cardiac signals where supraventricular extrasystoles with atrio-ventricular blocks (identified as pauses without a prematurity of the preceding QRS complex) can be seen.

The results of the validation of ICs categorization obtained using 55-channel clusters are summarized in table 1. For each gestational period and for each signal category, the number 
Fetus at 24 weeks

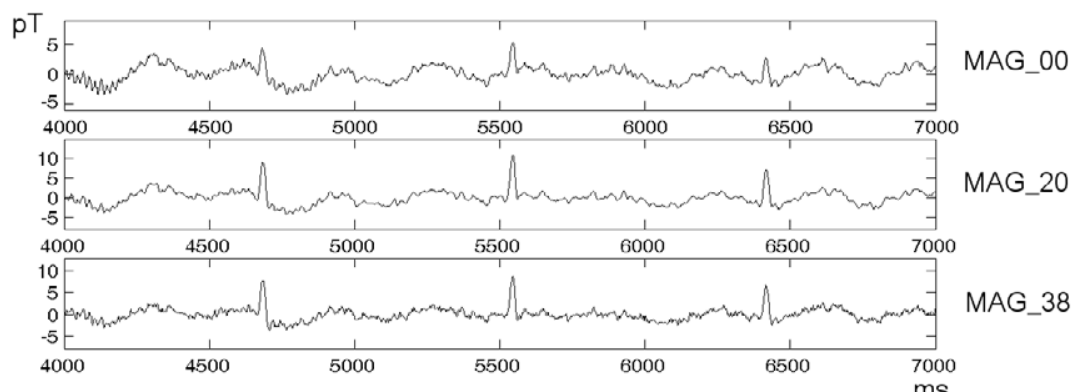

Fetus at 30 weeks

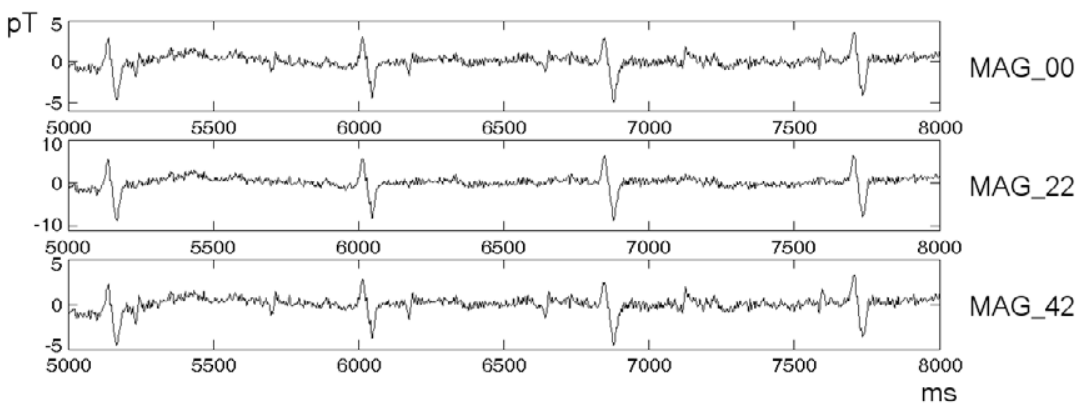

Fetus at 36 weeks

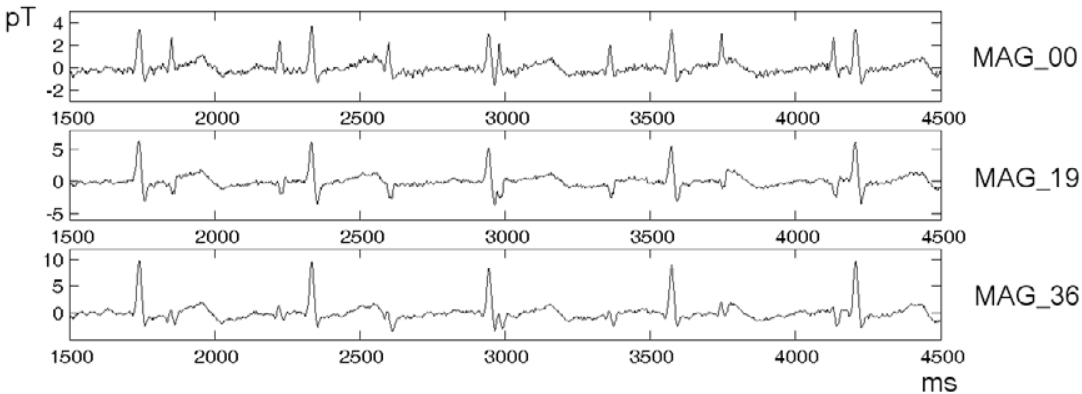

Arrhythmic fetus at 36 weeks

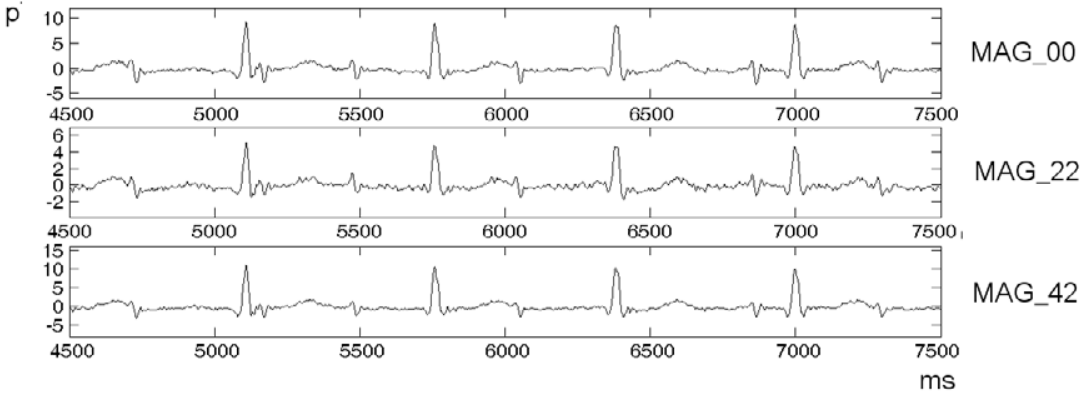

Figure 1. Examples of original fMCG recordings for three healthy fetuses $(24,30$ and 36 gestational weeks) and an arrhythmic fetus (36 gestational weeks). For each fetus, the recordings were selected in order to depict both positive and negative half spaces of the fetal magnetic field. The channel identifier is indicated. The intervals, given in ms, correspond to the time elapsed from the beginning of fMCG acquisition. Signal intensity is given in pT. 
Fetus at 24 weeks

19 channels

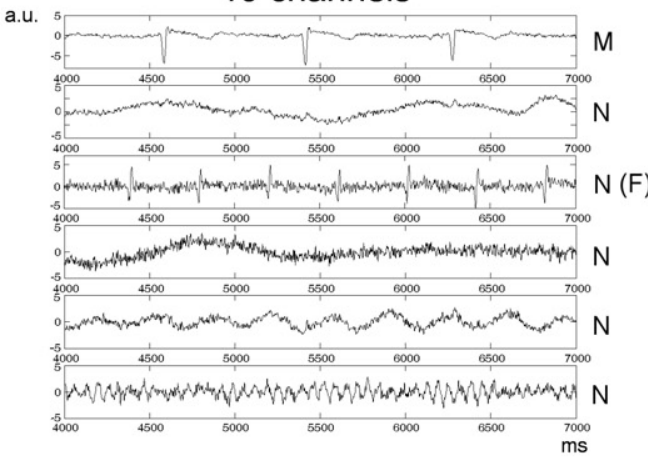

55 channels

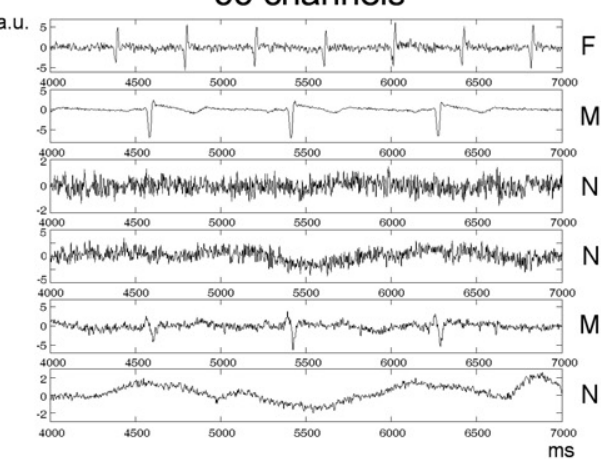

Fetus at 30 weeks

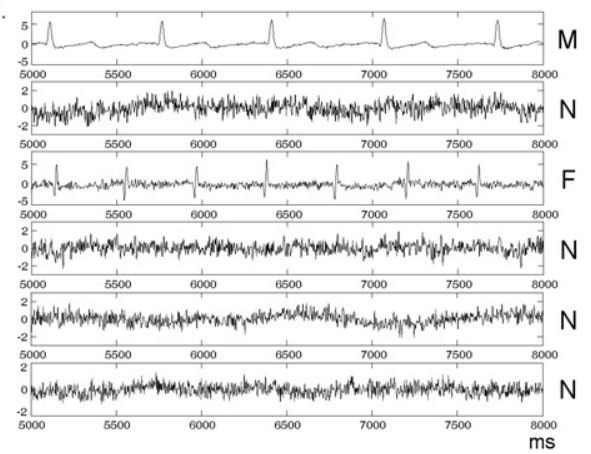

\section{5 channels}

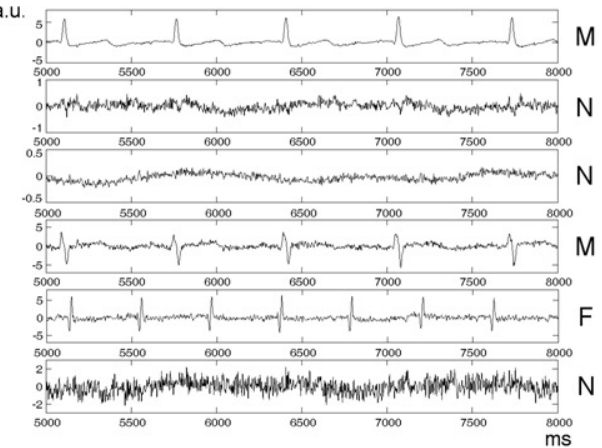

Fetus at 36 weeks

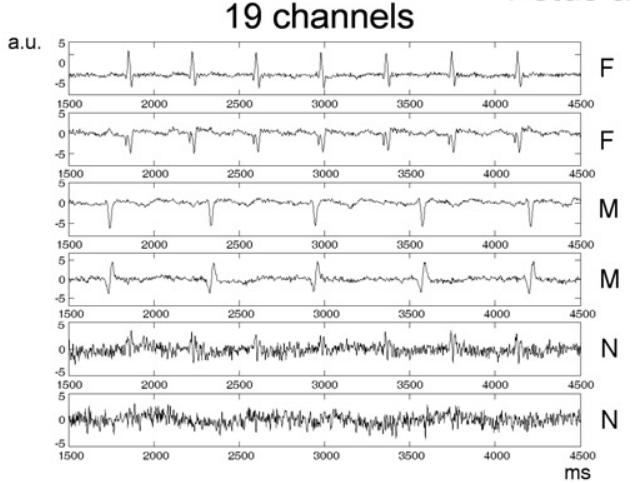

\section{5 channels}

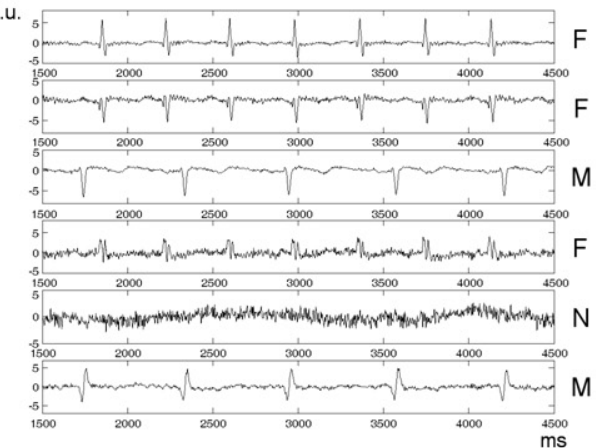

Figure 2. Intermediate output of the automatic method for the three healthy fetuses shown in figure 1. For each fetus, the six components separated using the 55-channel cluster (right) and one 19-channel cluster (left) are shown. The intervals, given in ms, are the same as the recordings shown in figure 1 . On the right of each drawing, the automatic classification is indicated $(\mathrm{F}=$ fetal, $\mathrm{M}=$ maternal, $\mathrm{N}=$ noise); in the case of discordant manual classification, the latter is put in parentheses next to the automatic one. Signal intensity is given in arbitrary units, because at this stage the correct signal amplitude has not been retrieved yet. 
Fetus at 24 weeks
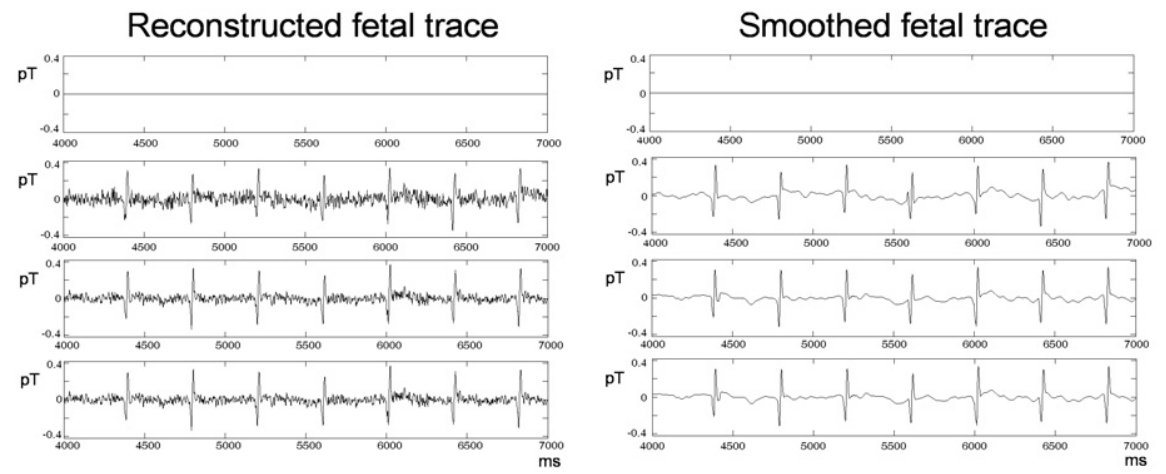

AUTOMATIC

19 channels

MANUAL

19 channels

AUTOMATIC

55 channels

MANUAL

55 channels

Fetus at 30 weeks
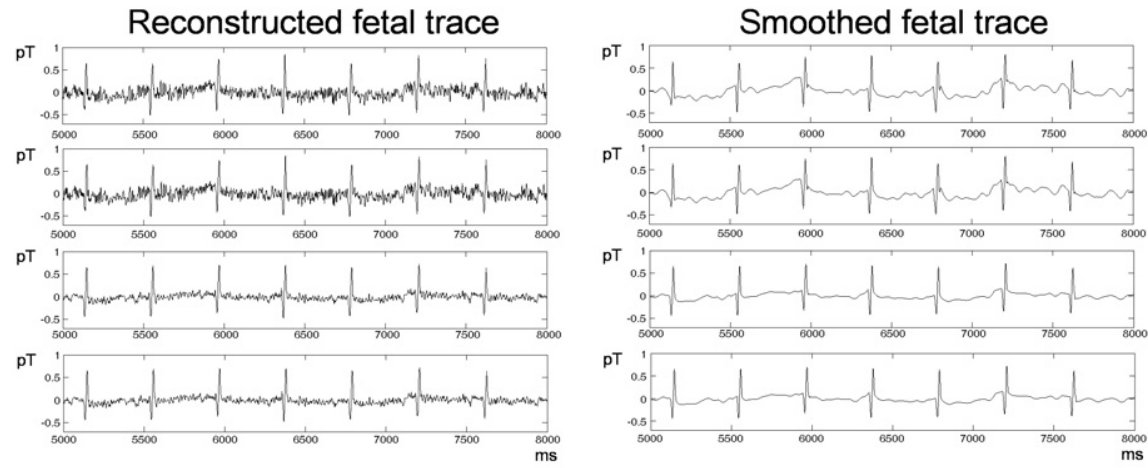

AUTOMATIC

19 channels

MANUAL

19 channels

AUTOMATIC

55 channels

MANUAL

55 channels

Fetus at 36 weeks

Reconstructed fetal trace

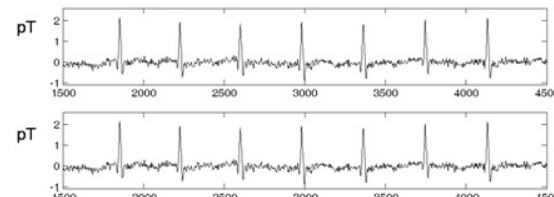

Smoothed fetal trace

$$
\text { PT }
$$

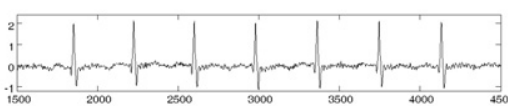

pT
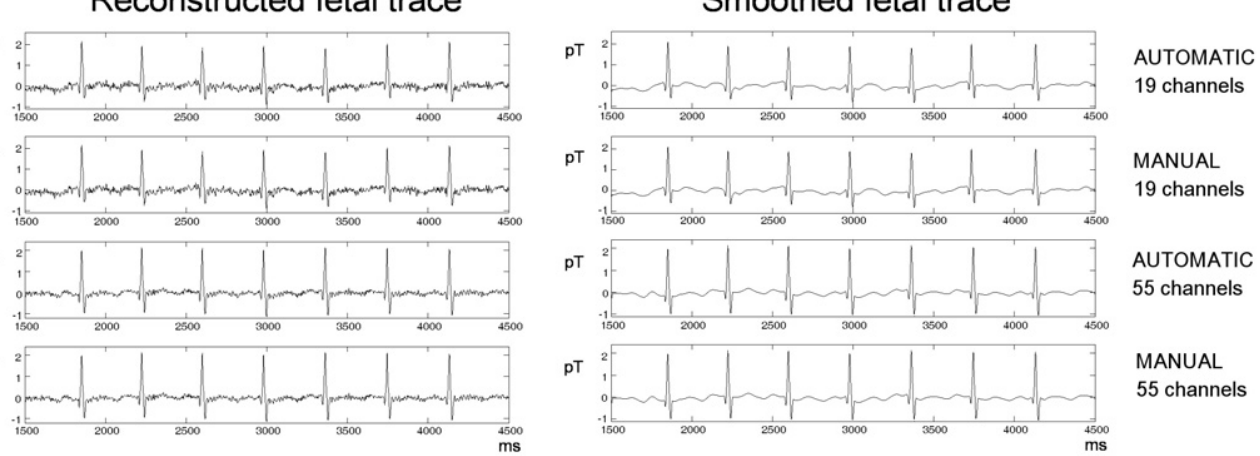

Figure 3. Fetal signals reconstructed from the components shown in figure 2. For each fetus, the raw and smoothed signals were obtained from the fetal components identified in figure 2 . Time is given in $\mathrm{ms}$, and the intervals correspond exactly to those of figure 2. Signal intensity is given in pT.

of components detected automatically $\left(N_{\text {tot }}\right)$ corresponded to those obtained manually $\left(N_{\operatorname{man}}\right)$, with the only exception of a fetal component (dataset at 24 weeks) that was wrongly classified as noise. In fact, the only true negatives and false positives values different from zero refer to the earliest gestational period.

Table 2 summarizes the same type of results obtained with 19-channel clusters. The reported figures demonstrate that the performances of the automatic method deteriorate with 


\section{Arrhythmic fetus at 36 weeks}

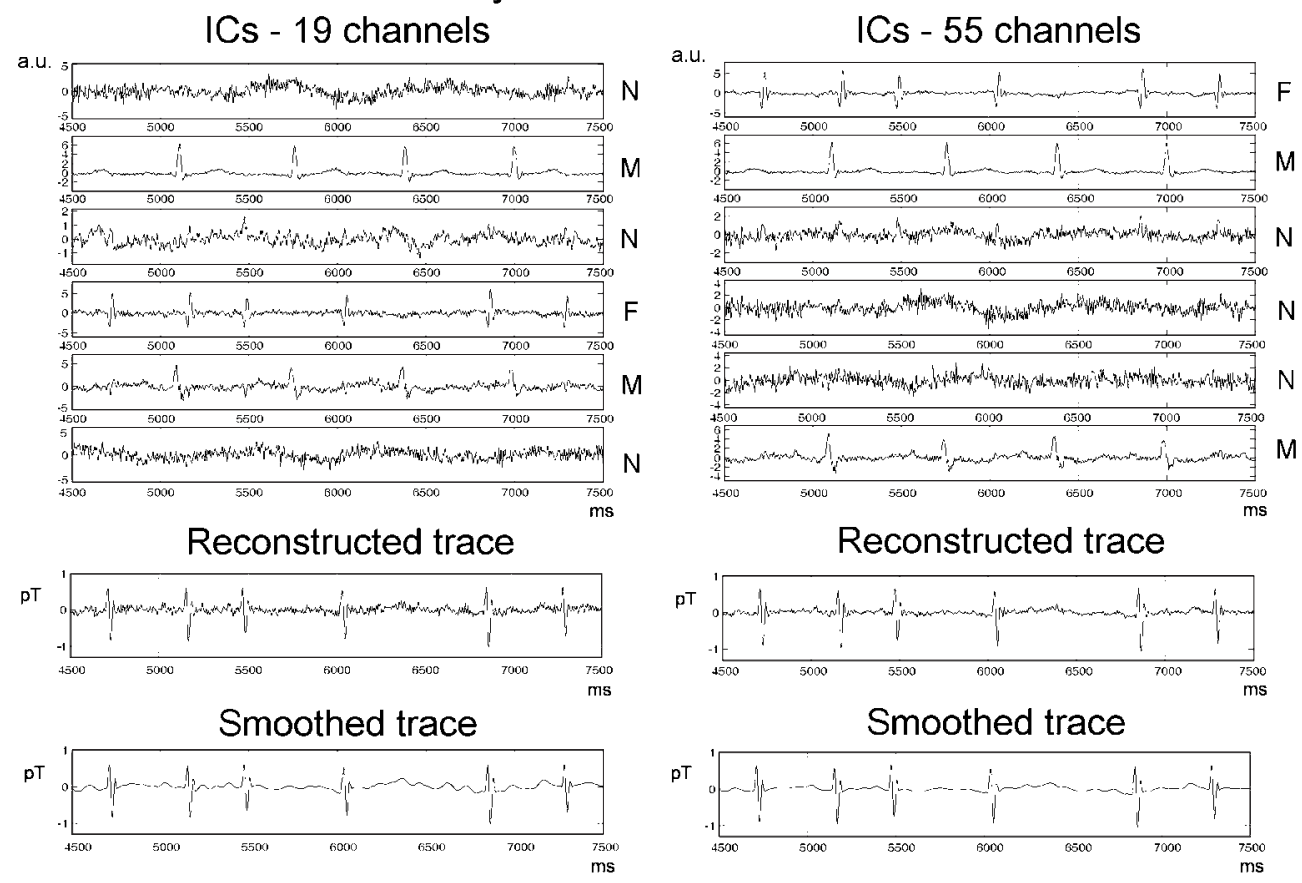

Figure 4. ICs and fetal traces obtained for the arrhythmic fetus shown in figure 1, using the 55-channel cluster (right) and one 19-channel cluster (left). The automatic classification is indicated on the right-hand side of each drawing. The reconstructed and smoothed fetal signals are shown below. Time is given in $\mathrm{ms}$, and the intervals correspond exactly to those of figure 1 . Signal intensity is given in $\mathrm{pT}$.

the use of smaller input clusters. As regards fetal components, true negative identifications (MIC) exist in all gestational periods, whereas false positive detections (ICIC) disappear only after the 31st week. All noise components were correctly detected from the 32nd gestational week onward, but false classifications of fetal components as maternal or noise occurred at all gestational ages.

Incorrect classification of fetal components as maternal ones occurred when not all fetal QRS complexes were detected because of residual large baseline shifts. Conversely, wrong classification of fetal components as noise occurred when the analysed component had not the required minimum SNR value: as a consequence, although very small fetal QRS complexes might perhaps be recognized by eye, those components were classified as noise. Two examples are provided in figure 2: the third component obtained from a 19-channel cluster for the fetus at 24 weeks had not the required SNR value and was classified as noise, while the corresponding component obtained with the 55-channel cluster (the first one) had a better SNR value and was correctly classified as fetal; the fifth component extracted for the fetus at 36 weeks using a 19-channel cluster presented fetal QRS complexes that were hardly detectable by eye: therefore, the automatic method classified it as noise, whereas the use of 55-channel cluster provided a better SNR, and the corresponding component (the forth) was classified correctly.

No validation of the reliability of the proposed automatic method could be performed for arrhythmic fetuses, because too few datasets were available. However, the results 
Table 1. Validation of ICs classification of components separated using clusters of 55 fMCG recordings. All findings refer to healthy fetuses and are grouped in gestational periods. For each signal category, the numbers $N_{\text {man }}, N_{\text {tot }}, N_{\text {corr }}$ and the per cent indices MIC and ICIC, respectively corresponding to true negatives and false positives, are provided.

\begin{tabular}{|c|c|c|c|c|c|c|}
\hline \multirow[b]{3}{*}{ Number of analysed components $\mathrm{s}^{\mathrm{a}}$} & \multicolumn{6}{|c|}{ Gestational period (weeks) } \\
\hline & $22-25$ & $26-28$ & $29-31$ & $32-34$ & $35-37$ & Total \\
\hline & 78 & 66 & 84 & 78 & 90 & 396 \\
\hline \multicolumn{7}{|l|}{ Manual $\left(N_{\operatorname{man}}\right)$} \\
\hline Fetal & 14 & 12 & 15 & 15 & 16 & 72 \\
\hline Maternal & 20 & 18 & 25 & 24 & 27 & 114 \\
\hline Noise & 44 & 36 & 44 & 39 & 47 & 210 \\
\hline \multicolumn{7}{|l|}{ Automatic $\left(N_{\text {tot }}\right)$} \\
\hline Fetal & 13 & 12 & 15 & 15 & 16 & 71 \\
\hline Maternal & 20 & 18 & 25 & 24 & 27 & 114 \\
\hline Noise & 45 & 36 & 44 & 39 & 47 & 211 \\
\hline \multicolumn{7}{|l|}{ Automatic $\left(N_{\text {corr }}\right)$} \\
\hline Fetal & 13 & 12 & 15 & 15 & 16 & 71 \\
\hline Maternal & 20 & 18 & 25 & 24 & 27 & 114 \\
\hline Noise & 44 & 36 & 44 & 39 & 47 & 210 \\
\hline \multicolumn{7}{|l|}{ MIC } \\
\hline Fetal & $7.1 \%$ & $0 \%$ & $0 \%$ & $0 \%$ & $0 \%$ & $1.4 \%$ \\
\hline Maternal & $0 \%$ & $0 \%$ & $0 \%$ & $0 \%$ & $0 \%$ & $0 \%$ \\
\hline Noise & $0 \%$ & $0 \%$ & $0 \%$ & $0 \%$ & $0 \%$ & $0 \%$ \\
\hline \multicolumn{7}{|l|}{ ICIC } \\
\hline Fetal & $0 \%$ & $0 \%$ & $0 \%$ & $0 \%$ & $0 \%$ & $0 \%$ \\
\hline Maternal & $0 \%$ & $0 \%$ & $0 \%$ & $0 \%$ & $0 \%$ & $0 \%$ \\
\hline Noise & $2.3 \%$ & $0 \%$ & $0 \%$ & $0 \%$ & $0 \%$ & $0.5 \%$ \\
\hline
\end{tabular}

${ }^{a}$ The number of analysed components is equal to the number of available datasets times six, because six ICs were extracted from each cluster.

obtained for the existing recordings with 55-channel and 19-channel clusters are summarized in table 3 .

The characteristics of the fetal traces obtained automatically and manually for all healthy fetuses are summarized in table 4 (55-channel clusters) and table 5 (19-channel clusters). The best results were achieved with the largest clusters, with a percentage of $85 \%$ reconstructed fetal signals with respect to the already available datasets for the earliest gestational period; from the 29th week onward, all fetal signals were restored. The manual reconstruction of fetal traces performed better than the automatic method only before the 26th week. In contrast, the number of fetal traces retrieved automatically with the use of smaller clusters was just $28 \%$ of the available clusters at early gestation, and it increased to the $84 \%$ only for the latest gestational period; in those cases, the manual retrieval of fetal signals performed was always better than the automatic method.

The average QRS amplitudes, standard deviations and SNR obtained for fetal traces reconstructed automatically and manually were always very similar when 55-channel clusters were used, independently of gestational age. Some larger differences were observed for the traces retrieved before the 29th week with 19-channel clusters, although this fact could partly be ascribed to the different detection rates, and, consequently, to the different number of fetal components available for signal reconstruction. However, when paired QRS differences were calculated for single fetuses, their absolute values were always less than $10 \mathrm{fT}$ for fetal signals restored from large clusters, and about $12 \mathrm{fT}$ for signals retrieved from small clusters. Average 
Table 2. Validation of ICs classification of components separated using clusters of $19 \mathrm{fMCG}$ recordings. All findings refer to healthy fetuses and are grouped in gestational periods. For each signal category, the numbers $N_{\text {man }}, N_{\text {tot }}, N_{\text {corr }}$ and the per cent indices MIC and ICIC, respectively corresponding to true negatives and false positives, are provided.

\begin{tabular}{lllllll}
\hline & \multicolumn{5}{c}{ Gestational period (weeks) } \\
& $22-25$ & $26-28$ & $29-31$ & $32-34$ & $35-37$ & Total \\
\cline { 2 - 7 } Number of analysed components ${ }^{\mathrm{a}}$ & 702 & 594 & 756 & 702 & 810 & 3564 \\
\hline Manual $\left(N_{\text {man }}\right)$ & & & & & & \\
$\quad$ Fetal & 87 & 80 & 147 & 154 & 169 & 637 \\
$\quad$ Maternal & 160 & 159 & 199 & 169 & 198 & 885 \\
$\quad$ Noise & 455 & 355 & 410 & 379 & 443 & 2042 \\
Automatic $\left(N_{\text {tot }}\right)$ & & & & & & \\
$\quad$ Fetal & 76 & 68 & 126 & 137 & 157 & 564 \\
$\quad$ Maternal & 163 & 164 & 203 & 169 & 198 & 897 \\
$\quad$ Noise & 463 & 362 & 427 & 396 & 455 & 2103 \\
Automatic $\left(N_{\text {corr }}\right)$ & & & & & & \\
Fetal & 73 & 68 & 123 & 137 & 157 & 558 \\
$\quad$ Maternal & 160 & 159 & 199 & 169 & 198 & 885 \\
$\quad$ Noise & 450 & 351 & 402 & 379 & 443 & 2025 \\
MIC & & & & & & \\
Fetal & $16.1 \%$ & $15.0 \%$ & $16.3 \%$ & $11.0 \%$ & $7.1 \%$ & $12.4 \%$ \\
$\quad$ Maternal & $0 \%$ & $0 \%$ & $0 \%$ & $0 \%$ & $0 \%$ & $0 \%$ \\
$\quad$ Noise & $1.1 \%$ & $1.1 \%$ & $2 \%$ & $0 \%$ & $0 \%$ & $0.8 \%$ \\
ICIC & & & & & & \\
$\quad$ Fetal & $3.4 \%$ & $0 \%$ & $2 \%$ & $0 \%$ & $0 \%$ & $0.9 \%$ \\
$\quad$ Maternal & $1.9 \%$ & $3.1 \%$ & $2.1 \%$ & $0 \%$ & $0 \%$ & $1.4 \%$ \\
$\quad$ Noise & $2.9 \%$ & $3.1 \%$ & $6.1 \%$ & $4.5 \%$ & $2.7 \%$ & $3.8 \%$ \\
\hline S & & &
\end{tabular}

${ }^{a}$ Since data refer to the analysis of components separated with 19-channel clusters, the total number of analysed components is equal to the number of analysed components given in table 1 times 9 (number of small clusters for each dataset).

noise amplitudes in fMCG recordings, their standard deviations and ranges are provided to allow a comparison with average QRS intensities and to justify the attained SNR values.

\section{Discussion}

A completely automatic method for the reconstruction of fetal signals from fMCG recordings would be extremely valuable for the prenatal monitoring of cardiac function, and the results of our study suggest that the proposed method might be effective. However, the robustness and reliability of a new method are important aspects to be carefully evaluated.

The first feature to be assessed was the ability of ICA to separate all source components preserving distinct signal morphology and sufficient SNR: this was the essential prerequisite to achieve an accurate classification of components and a consequent reliable reconstruction of fetal signals. In fact, the outcomes of our validation study confirmed that the performances of the automatic classification of components, while only partly dependent on gestational age and noise, were mainly affected by clusters' dimensions: this was not surprising, since ICA uses neural networks and performs better with large input datasets (Comani et al 2004a).

As a matter of fact, in the validation study performed on healthy fetuses, the use of 55-channel clusters ensured extremely high success rates in the automatic categorization of components, which might be ascribed to the achieved high SNR and to the absence of 
Table 3. Validation of ICs classification for arrhythmic fetuses. ICs were separated using clusters of 55 and 19 fMCG recordings. For each fetus, the numbers $N_{\text {man }}, N_{\text {tot }}, N_{\text {corr }}$ are provided. The per cent indices MIC and ICIC, respectively corresponding to true negatives and false positives, are given only for the analysis of the output of 19-channels clusters, because they were null for all fetuses when the 55-channel clusters were used.

\begin{tabular}{|c|c|c|c|c|}
\hline Gestational age (weeks) & $\begin{array}{l}\text { Fetus no. } 1 \\
30\end{array}$ & $\begin{array}{l}\text { Fetus no. } 2 \\
32\end{array}$ & $\begin{array}{l}\text { Fetus no. } 3 \\
36\end{array}$ & $\begin{array}{l}\text { Fetus no. } 4 \\
37\end{array}$ \\
\hline $\begin{array}{l}\text { Total number of ICs } \\
\text { (1 cluster of } 55 \text { traces) }\end{array}$ & 6 & 6 & 6 & 6 \\
\hline \multicolumn{5}{|l|}{ Manual $\left(N_{\operatorname{man}}\right)$} \\
\hline Fetal & 2 & 1 & 1 & 1 \\
\hline Maternal & 3 & 2 & 2 & 1 \\
\hline Noise & 1 & 3 & 3 & 4 \\
\hline \multicolumn{5}{|l|}{ Automatic $\left(N_{\text {tot }}\right)$} \\
\hline Fetal & 2 & 1 & 1 & 1 \\
\hline Maternal & 3 & 2 & 2 & 1 \\
\hline Noise & 1 & 3 & 3 & 4 \\
\hline \multicolumn{5}{|l|}{ Automatic $\left(N_{\text {corr }}\right)$} \\
\hline Fetal & 2 & 1 & 1 & 1 \\
\hline Maternal & 3 & 2 & 2 & 1 \\
\hline Noise & 1 & 3 & 3 & 4 \\
\hline $\begin{array}{l}\text { Total number of ICs } \\
\text { (nine clusters of } 19 \text { traces) }\end{array}$ & 54 & 54 & 54 & 54 \\
\hline \multicolumn{5}{|l|}{ Manual $\left(N_{\operatorname{man}}\right)$} \\
\hline Fetal & 3 & 7 & 8 & 15 \\
\hline Maternal & 23 & 12 & 12 & 12 \\
\hline Noise & 28 & 35 & 34 & 17 \\
\hline \multicolumn{5}{|l|}{ Automatic $\left(N_{\text {tot }}\right)$} \\
\hline Fetal & 2 & 3 & 6 & 13 \\
\hline Maternal & 23 & 12 & 12 & 12 \\
\hline Noise & 29 & 39 & 36 & 19 \\
\hline \multicolumn{5}{|l|}{ Automatic $\left(N_{\text {corr }}\right)$} \\
\hline Fetal & 2 & 3 & 6 & 13 \\
\hline Maternal & 23 & 12 & 12 & 12 \\
\hline Noise & 28 & 35 & 34 & 17 \\
\hline \multicolumn{5}{|l|}{ MIC } \\
\hline Fetal & $33.3 \%$ & $57.1 \%$ & $25 \%$ & $13.3 \%$ \\
\hline Maternal & $0 \%$ & $0 \%$ & $0 \%$ & $0 \%$ \\
\hline Noise & $0 \%$ & $0 \%$ & $0 \%$ & $0 \%$ \\
\hline \multicolumn{5}{|l|}{ ICIC } \\
\hline Fetal & $0 \%$ & $0 \%$ & $0 \%$ & $0 \%$ \\
\hline Maternal & $0 \%$ & $0 \%$ & $0 \%$ & $0 \%$ \\
\hline Noise & $3.6 \%$ & $11.4 \%$ & $5.9 \%$ & $11.8 \%$ \\
\hline
\end{tabular}

contamination among source signals (figure 2). Those conditions were also attained during early pregnancy, and only one fetal component, out of 396 analysed ones, was incorrectly classified as noise. It was the case of a fetus at 24 weeks, for which the mother referred strong movements during acquisition; they most likely caused the persistence of some noise and baseline drifts in all separated components, also hampering the manual detection of components. The use of 55-channel clusters was also extremely effective in the analysis of arrhythmic fetuses datasets, with no wrong component classification (see table 3). 
Table 4. Characteristics of fetal traces retrieved using clusters of $55 \mathrm{fMCG}$ recordings. All findings are grouped in gestational periods. The number of reconstructed fetal traces is also given as the per cent value with respect to available fMCG clusters (in parentheses). The amplitude of all signals (average noise, its range and related standard deviations, average QRS amplitudes and related standard deviations) are given in fT; SNR is expressed in $\mathrm{dB}$.

\begin{tabular}{|c|c|c|c|c|c|c|}
\hline & \multicolumn{6}{|c|}{ Gestational period (weeks) } \\
\hline & $22-25$ & $26-28$ & $29-31$ & $32-34$ & $35-37$ & $22-37$ \\
\hline $\begin{array}{l}\text { Average noise in raw } \\
\text { fMCG recordings (SD) }\end{array}$ & $597(240)$ & $497(91)$ & $354(115)$ & $419(155)$ & $434(201)$ & $478(188)$ \\
\hline Noise range in raw traces & $131-1607$ & $106-1467$ & $145-1258$ & $124-1265$ & $95-1610$ & $95-1610$ \\
\hline Number of 55-channel clusters & 13 & 11 & 14 & 13 & 15 & 66 \\
\hline \multicolumn{7}{|l|}{ Automatic method } \\
\hline $\begin{array}{l}\text { Number of reconstructed } \\
\text { fetal traces }{ }^{\mathrm{a}}\end{array}$ & $11(85 \%)$ & $10(91 \%)$ & $14(100 \%)$ & $13(100 \%)$ & $15(100 \%)$ & $63(95 \%)$ \\
\hline Average QRS amplitude (SD) & $659(488)$ & $879(560)$ & $958(336)$ & $1236(911)$ & $2201(597)$ & $1246(579)$ \\
\hline Average SNR & 10.3 & 11.1 & 11.3 & 11.9 & 16.8 & 12.0 \\
\hline \multicolumn{7}{|l|}{ Manual method } \\
\hline $\begin{array}{l}\text { Number of reconstructed } \\
\text { fetal traces }{ }^{\mathrm{a}}\end{array}$ & $12(92 \%)$ & $10(91 \%)$ & $14(100 \%)$ & $13(100 \%)$ & $15(100 \%)$ & $64(97 \%)$ \\
\hline Average QRS amplitude (SD) & $660(489)$ & $878(562)$ & $956(339)$ & $1235(911)$ & $2198(597)$ & $1245(580)$ \\
\hline Average SNR & 10.0 & 11.1 & 11.3 & 11.9 & 16.8 & 12.0 \\
\hline $\begin{array}{l}\text { Per cent QRS difference } \\
\text { Range of real QRS differences }\end{array}$ & $\begin{array}{l}0.21 \% \\
(-9,9)\end{array}$ & $\begin{array}{l}0.13 \% \\
(-9,5)\end{array}$ & $\begin{array}{l}0.33 \% \\
(-7,9)\end{array}$ & $\begin{array}{l}0.23 \% \\
(-8,6)\end{array}$ & $\begin{array}{l}0.13 \% \\
(-6,10)\end{array}$ & $\begin{array}{l}0.21 \% \\
(-9,10)\end{array}$ \\
\hline
\end{tabular}

${ }^{a}$ In parentheses: the per cent value of reconstructed fetal traces with respect to the number of available clusters.

b The difference between the average QRS amplitudes in the fetal traces reconstructed automatically and manually is calculated for each fetus and normalized with respect to the average QRS amplitude obtained with the manual procedure; the per cent value is calculated for each fetus and the group average is provided in the table.

${ }^{c}$ The values given in parentheses correspond to the minimum and maximum real QRS differences, given in fT, detected among all fetuses in a group.

Conversely, the categorization of components separated with 19-channel clusters was much less satisfactory for both healthy and arrhythmic fetuses. Although we previously demonstrated that clusters of this dimension were sufficient to obtain stable and repeatable ICA outputs (Comani et al 2004a), the relative peak-to-peak amplitudes of fetal signals and background noise in input recordings, in combination with the low number of input channels, determined the low SNR values and the small maternal interference observed in some cases. Consequently, a fairly large number of fetal components were missed (from $7.1 \%$ to $16.3 \%$ ), with a percentage of fetal components erroneously classified as noise ranging from $2.7 \%$ to $6.1 \%$; no relation with gestational age was observed. Cases of fetal components that were categorized as maternal occurred before the $32 \mathrm{nd}$ week, most probably because some residual trace instability, which was due to fetal movements or maternal respiration, prevented the automatic recognition of all fetal QRS complexes. The percentage of wrong classifications was even larger when dealing with arrhythmic fetuses (see table 3).

When analysing the characteristics of the fetal signals reconstructed with the two methods, the dependency of the automatic method effectiveness on the dimension of the input cluster was confirmed. In fact, the results related to 55-channel clusters showed extremely high success rates in the reconstruction of fetal traces with a high SNR, particularly remarkable for early gestation, when the average fetal QRS amplitude is comparable to average background noise. As regards signal intensity, the reliability of the automatic model was supported 
Table 5. Characteristics of fetal traces retrieved using clusters of 19 fMCG recordings. All findings are grouped in gestational periods. The number of reconstructed fetal traces is given also as the per cent value with respect to available fMCG clusters (in parentheses). The amplitude of all signals (average noise, its range and related standard deviations, average QRS amplitudes and related standard deviations) are given in $\mathrm{fT}$; SNR is expressed in $\mathrm{dB}$.

\begin{tabular}{|c|c|c|c|c|c|c|}
\hline & \multicolumn{6}{|c|}{ Gestational period (weeks) } \\
\hline & $22-25$ & $26-28$ & $29-31$ & $32-34$ & $35-37$ & $22-37$ \\
\hline $\begin{array}{l}\text { Average noise in raw } \\
\text { fMCG recordings (SD) }\end{array}$ & $597(240)$ & $497(91)$ & $354(115)$ & $419(155)$ & $434(201)$ & $478(188)$ \\
\hline Noise range in raw traces & $131-1607$ & $106-1467$ & $145-1258$ & $124-1265$ & $95-1610$ & $95-1610$ \\
\hline Number of 19-channel clusters & 117 & 99 & 126 & 117 & 135 & 594 \\
\hline \multicolumn{7}{|l|}{ Automatic method } \\
\hline $\begin{array}{l}\text { Number of reconstructed } \\
\text { fetal traces }{ }^{\mathrm{a}}\end{array}$ & $33(28 \%)$ & $32(32 \%)$ & $63(50 \%)$ & $65(56 \%)$ & $113(84 \%)$ & $306(52 \%)$ \\
\hline Average QRS amplitude (SD) & $569(468)$ & $827(568)$ & $1036(273)$ & $1242(999)$ & $2106(657)$ & $1403(621)$ \\
\hline SNR & 8.1 & 9.4 & 8.8 & 9.7 & 12.2 & 9.6 \\
\hline \multicolumn{7}{|l|}{ Manual method } \\
\hline $\begin{array}{l}\text { Number of reconstructed } \\
\text { fetal traces }{ }^{\text {a }}\end{array}$ & $45(38 \%)$ & $39(39 \%)$ & $72(57 \%)$ & $67(57 \%)$ & $120(89 \%)$ & $343(58 \%)$ \\
\hline Average QRS amplitude (SD) & $533(429)$ & $776(560)$ & $1041(277)$ & $1239(998)$ & $2101(658)$ & $1392(617)$ \\
\hline SNR & 7.6 & 8.6 & 8.6 & 9.5 & 12.1 & 9.1 \\
\hline $\begin{array}{l}\text { Per cent QRS difference } \\
\text { Range of real QRS differences }\end{array}$ & $\begin{array}{l}-0.19 \% \\
(-10,6)\end{array}$ & $\begin{array}{l}0.63 \% \\
(-12,5)\end{array}$ & $\begin{array}{l}-0.39 \% \\
\quad(-23,8)^{\mathrm{d}}\end{array}$ & $\begin{array}{l}0.48 \% \\
(-7,9)\end{array}$ & $\begin{array}{l}0.25 \% \\
(-6,11)\end{array}$ & $\begin{array}{l}0.16 \% \\
(-23,11)^{\mathrm{d}}\end{array}$ \\
\hline
\end{tabular}

${ }^{a}$ In parentheses: the per cent value of reconstructed fetal traces with respect to the number of available clusters.

b The difference between the average QRS amplitudes in the fetal traces reconstructed automatically and manually is calculated for each fetus and normalized with respect to the average QRS amplitude obtained with the manual procedure; the per cent value is calculated for each fetus and the group average is provided in the table.

${ }^{c}$ The values given in parentheses correspond to the minimum and maximum real QRS differences, given in fT, detected among all fetuses in a group.

d The lower limit in this range is an outlier that refers to a fetus for which the automatic method detected one fetal component instead of two, which were used, in contrast, for the manual reconstruction of the signal.

by the negligible real differences, always less than $10 \mathrm{fT}$ in absolute value, which were observed between the amplitudes of paired QRS complexes in fetal traces retrieved with the automatic and manual procedures. Concerning signal morphology, we already demonstrated the reliability of ICA to reconstruct source signals maintaining their original waveform (Comani et al 2004d, 2004e).

Similar results were obtained for the fetal traces reconstructed from 19-channel clusters, but detection rates were much poorer, SNR was generally lower, and amplitude differences of paired QRS complexes were rather larger; it is worth noting that average QRS amplitudes, calculated from traces reconstructed with the automatic and manual procedures, were comparable from the 29th week onward, as were the ranges of paired real QRS differences.

Pertaining to arrhythmic fetuses, the same outcomes were found for the four available datasets as to signal morphology and intensity.

Finally, some inconsistencies may be observed between the detection rates reported in tables 4 and 5 and the true negative and false positive values given in tables 1 and 2, but they can be explained if we consider that detection rates are calculated with respect to available fMCG datasets, among which some particularly noisy recordings or extremely weak fetal signals may occur, hampering the identification of fetal components even for a skilled investigator. On 
the other hand, true negative and false positive values are calculated with respect to manual components' detection, and not to available fMCG datasets; for this reason, those figures may seem, but are not, inconsistent.

\section{Conclusion}

Different MCG devices are used in research laboratories and clinical environments, each with a specific sensor configuration and technical characteristics; moreover, a great effort is presently being made to develop new MCG devices dedicated to fetal acquisitions and/or suitable to work in a hospital setting and portable bedside (Lowery et al 2003, Robinson et al 2001, Budnyk et al 2004, Nomura et al 2004, Suzuki et al 2004, Brisinda et al 2005). For this reason, the proposed model was implemented in an open architecture software suitable to process data recorded with different MCG systems. Once datasets from those devices and more numerous fMCG recordings for arrhythmic fetuses are available for analysis, we will further test our method to draw comprehensive conclusions on its effectiveness and consistency. However, the results already obtained for healthy and arrhythmic fetuses seem to indicate that the use of large input clusters can provide reliable reconstructions of fetal signals also for early gestation and/or from fMCG recordings with a fairly low SNR, and that the proposed model can be considered a first step forward in the standardization of the procedure for the extraction of fetal cardiac signals from fMCG.

\section{References}

Abraham-Fuchs K, Härer W, Schneider S and Stefan H 1990 Pattern recognition in biomagnetic signals by spatiotemporal correlation and application to the localisation of propagating neuronal activity Med.Biol. Eng. Comput. 28 398-406

Brisinda D et al 2005 Multichannel mapping of fetal magnetocardiogram in an unshielded hospital setting Prenat. Diagn. 25 376-82

Budnyk M M et al 2004 Low-cost 7-channel magnetocardiographic system for unshielded environment Proc. 14th Int. Conf. on Biomagnetism (Boston, MA, 8-12 Aug.) pp 617-8

Chen M, Wakai R T and Van Veen B 2001 Eigenvector based spatial filtering of fetal biomagnetic signals J. Perinat. Med. 29 486-96

Comon P 1994 Independent component analysis_a new concept? Signal Process 36 287-314

Comani S et al 2004a Independent component analysis: fetal signal reconstruction from magnetocardiographic recordings Comput. Methods Programs Biomed. 75 163-77

Comani S et al 2004b Integrated software suite for magnetocardiographic data analysis: a proposal based on interactive programming environment Methods. Inf. Med. 44 114-23

Comani S et al 2004c Characterization of fetal arrhythmias by means of fetal magnetocardiography in three cases of difficult ultrasonographic imaging Pacing Clin. Electrophysiol. 27 1647-55

Comani S, Mantini D, Alleva G, Di Luzio S and Romani G L 2004d Fetal magnetocardiographic mapping using independent component analysis Physiol. Meas. 25 1459-72

Comani S, Mantini D, Lagatta A, Esposito F, Di Luzio S and Romani G L 2004e Time course reconstruction of fetal cardiac signals from fMCG: independent component analysis vs. adaptive maternal beat subtraction Physiol. Meas. 25 1305-21

Comani S, Liberati M, Mantini D, Merlino B, Alleva G, Gabriele E, Di Luzio S and Romani G L 2005a Beat-to-beat estimate of fetal cardiac time intervals using magnetocardiography: longitudinal charts of normality ranges and individual trends Acta Obstet. Gynecol. Scand. at press

Comani S, Mantini D, Alleva G, Gabriele E, Liberati M and Romani G L 2005b Simultaneous monitoring of separate fetal magnetocardiographic signals in twin pregnancy Physiol. Meas. 26 193-201

Della Penna S et al 2000 Biomagnetic systems for clinical use Phil. Mag. 80 937-48

Esposito F, Scarabino T, Hyvarinen A, Himberg J, Formisano E, Comani S, Tedeschi G, Goebel R, Seifritz E and Di Salle F 2005 Independent component analysis of fMRI group studies by self-organizing clustering Neuroimage 25 193-205 
Grimm B, Kähler C, Schleussner E, Schneider U, Haueisen J and Seewald H J 2003 Influence of intrauterine growth restriction on cardiac time intervals evaluated by fetal magnetocardiography Early Hum. Dev. 74 1-11

Horigome $\mathrm{H}$ et al 2001 Detection of cardiac hypertrophy in the fetus by approximation of the current dipole using magnetocardiography Pediatr. Res. 50 242-5

Hosono T, Kanegawa T, Chiba Y, Kandori A and Tsukada K 2002 The coincidence of fetal magnetocardiography and direct electrocardiography in a case of fetal atrial flutter due to intracardiac tumor Fetal Diagn. Ther. 17 331-3

Hyvärinen A 1998 New approximations of differential entropy for independent component analysis and projection pursuit Adv. Neural Inf. Process. Syst. $10273-9$

Hyvärinen A 1999 Fast and robust fixed-point algorithms for independent component analysis IEEE. Trans. Neural Netw. 10 626-34

Hyvärinen A and Oja E 2000 Independent component analysis: algorithms and applications Neural Netw. 13 411-30

James C J and Hesse C W 2005 Independent component analysis for biomedical signals Physiol. Meas. 26 R15-39

Kähler C et al 2001 The application of fetal magnetocardiography (FMCG) to investigate fetal arrhythmias and congenital heart defects (CHD) Prenat. Diag. 21 176-82

Kandori A et al 2003 Classifying cases of fetal Wolff-Parkinson-White syndrome by estimating the accessory pathway from fetal magnetocardiograms Med. Biol. Eng. Comput. 41 33-9

Kariniemi V, Ahopelto J, Karp P J and Katila T E 1974 The fetal magnetocardiogram J. Perinat. Med. 2 214-6

Lewis M J 2003 Review of electromagnetic source investigations of the fetal heart Med. Eng. Phys. 25 801-10

Li Z, Strasburger J F, Cuneo B F, Gotteiner N L and Wakai R T 2004 Giant fetal magnetocardiogram P waves in congenital atrioventricular block: a marker of cardiovascular compensation? Circulation 110 2097-101

Lowery C L et al 2003 Noninvasive antepartum recording of fetal S-T segment with a newly developed 151-channel magnetic sensor system Amer. J. Obstet. Gynecol. 188 1491-7

Mantini D et al 2004 Tailoring of the independent component analysis to multi-channel fMCG recordings for an optimal reconstruction of the fetal cardiac signal Biomed. Tech. 48 186-8

Mitra S K and Kaiser F 1993 Handbook for Digital Signal Processing (New York: Wiley)

Nakamura H, Yoshida M, Kotani M, Akazawa K and Moritani T J 2004 The application of independent component analysis to the multi-channel surface electromyographic signals for separation of motor unit action potential trains: part II-modelling interpretation Electromyogr. Kinesiol. $14433-41$

Nomura M, Nakaya Y, Ito S and Itozaki H 2004 Visualization of cardiac electrical current using 32-channel high temperature superconducting quantum interference device Proc. 14th Int. Conf. on Biomagnetism (Boston, MA, 8-12 Aug.) pp 632-33

Pichler A and Sowa M G 2005 Blind source separation of photoacoustic depth profiles into independent components Appl. Spectrosc. 59 164-72

Robinson S E et al 2001 A biomagnetic instrument for human reproductive assessment Proc. 12th Int. Conf. on Biomagnetism (Helsinki University of Technology, Espoo, Finland, 13-17 Aug. 2000) ed J Nenonen et al pp 919-22

Salustri C and Kronberg E 2004 Language-related brain activity revealed by independent component analysis Clin. Neurophysiol. $115385-95$

Saidi S A, Holland C M, Kreil D P, MacKay D J, Charnock-Jones D S, Print C G and Smith S K 2004 Independent component analysis of microarray data in the study of endometrial cancer Oncogene 23 6677-83

Stinstra J G and Peters M J 2002 The influence of fetoabdominal tissues on fetal ECGs and MCGs Arch Physiol. Biochem. 110 165-76

Stinstra J G, Peters M J and Quartero H W P 2000 Extracting reliable data from the fetal MCG Proc. Int. Conf. on Biomagnetism 591-4

Stinstra J et al 2002 Multicentre study of fetal cardiac time intervals using magnetocardiography Brit. J. Obstr Gynecol. 109 1235-43

Suzuki D et al 2004 Development of multi-channel compact HTS MCG system using transversal magnetically shielded cylinder Proc. 14th Int. Conf. on Biomagnetism (Boston, MA, 8-12 Aug.) p 662

Taylor M J et al 2003 Non-invasive fetal electrocardiography in singleton and multiple pregnancies Br. J. Obstet. Gynaecol. 110 668-78

Tran Y, Craig A, Boord P and Craig D 2004 Using independent component analysis to remove artifact from electroencephalographic measured during stuttered speech Med .Biol. Eng. Comput. 42 627-33

van Leeuwen P, Hailer B, Bader W, Geissler J, Trowitzsch E and Gronemeyer D H 1999 Magnetocardiography in the diagnosis of fetal arrhythmia Br. J. Obstet. Gynaecol. 106 1200-8

van Leeuwen P, Lange S, Klein A, Geue D, Zhang Y, Krause H J and Gronemeyer D 2004 Reproducibility and reliability of fetal cardiac time intervals using magnetocardiography Physiol. Meas. 25 539-52 
Vrba J, Robinson S E, McCubbin J, Lowery C L, Eswaran H, Wilson J D, Murphy P and Preissl H 2004 Fetal MEG redistribution by projection operators IEEE. Trans. Biomed. Eng. 51 1207-18

Wakai R T 2004 Assessment of fetal neurodevelopment via fetal magnetocardiography Exp. Neurol. 190 S65-71

Wakai R T, Lengle J M and Leuthold A C 2000 Transmission of electric and magnetic foetal cardiac signals in a case of ectopia cordis: the dominant role of the vernix caseosa Phys. Med. Biol. 45 1989-95

Wakai R T, Strasburger J F, Li Z, Deal B J and Gotteiner N L 2003 Magnetocardiographic rhythm patterns at initiation and termination of fetal supraventricular tachycardia Circulation 107 307-12

Zhou W and Gotman J 2005 Removing eye-movement artifacts from the EEG during the intracarotid amobarbital procedure Epilepsia 46 409-14 